\title{
An on-line interpretive Rorschach approach: Using Exner's comprehensive system
}

\author{
WILLIAM G. HARRIS, DAWN NIEDNER, CRAIG FELDMAN, \\ ANDREA FINK, and JAMES H. JOHNSON \\ Illinois Institute of Technology, Chicago, Illinois 60616
}

\begin{abstract}
This paper describes the development of an on-line interactive Rorschach interpretation method for the Exner comprehensive system. The findings of a study to examine the validity of the computer algorithm are reported. These findings suggest that the automated algorithm is able to provide an output report similar in quality to that of an expert clinician. The major strengths of the system were in assessing the person's psychological state and aiding in the formulation of diagnostic understanding and treatment plans. The major weakness was its limited description of the adaptive capabilities and interpersonal qualities of the person.
\end{abstract}

Two important innovations in the area of psychological assessment in the last decade were the use of on-line computer technology and the introduction of the comprehensive Rorschach system (Exner, 1974). Although the contributions of these two innovations were quite different, both were responsible for refining aspects of our understanding and knowledge of psychological assessment.

The use of the computer in psychological assessment has focused primarily on nonprojective measures. These psychological tools traditionally have used standardized administration and scoring procedures and prespecified interpretive rules. Projective techniques have not attracted the same attention. The lack of computerized applications of projective techniques can be explained by reasons including: (1) the complex administration of projective methods, (2) the loosely defined interpretive rules, and (3) the resistance of clinicians to computerassisted psychological evaluations.

Many of the projective techniques, such as the Rorschach and TAT, entail verbatim response recording and open-ended inquiries. These assessment tools generate a wealth of information for each test stimulus, which makes computerized administration and scoring highly undesirable. Adding to this difficulty are the numerous approaches to administering, scoring, and interpreting projective data. Moreover, the interpretive rules for these measures tend to be less well-defined than nonprojective measures. Many of the interpretive rules also lack adequate empirical support. Such concerns are further compounded by clinicians' reluctance to accept computer-assisted projective methods. Some clinicians insist that the computerization of projective tools would sacrifice the idiography of the individual's personality. However, even though automation of projective tools is likely to result in some loss of idiographic information,

Requests for reprints should be sent to William G. Harris, Department of Psychology, Illinois Institute of Technology, Chicago, Illinois 60616. it may offer a more systematic way of interpreting projective data.

To date, full automation of projective methods has not been shown to be clinically or economically feasible. Efforts to adapt projective techniques to the computer medium have centered on developing interpretation and report methods. Veldman (1967) and Veldman, Menaker, and Peck (1969), using a sentence-completion test, designed an automated interpretive system. Smith (1968) constructed a computer-generated method for the TAT. Gorham (1967) developed a computer-scoring system for the Holtzman inkblot technique. Unfortunately, further development of these interpretive systems has not been actively pursued. One of the more ambitious efforts in this area has been the computerassisted interpretation of Piotrowski's (1974) Rorschach system. The rationale for applying computer technology to his perceptanalysis procedure was the large volume of data and the numerous interpretive rules required to analyze an individual protocol. Since its introduction, the computerized perceptanalysis Rorschach system (currently known as the Piotrowski Automated Rorschach, or PAR) has undergone three major revisions and has more than 900 rules (Piotrowski, 1980).

Briefly, the PAR approach requires that a trained psy. chologist code each Rorschach response of the protocol into a string of computer-defined variables. This coded information is then entered into the computer for processing. The coding method used maintains many of the idiographic features of the Rorschach data. Piotrowski (1980) points out that the PAR system "does not rely on group statistics for discovery of relevant and meaningful test components, and/or for unravelling the mean ing of the basic components of the test responses" (p. 87). However, a salient problem for the PAR is its underutilization by clinicians. Based on a recent survey, few psychologists are well trained in the Piotrowski method of Rorschach analysis (Exner, 1976). The intricate scoring system of the PAR seems to be a key reason for its underutilization, even though reliability and 
validity studies have yielded promising findings. Nonetheless, the PAR demonstrated that a meaningful algorithm for computer use can be developed for projective measures.

The purpose of this paper is to describe the development of a microcomputer-based Rorschach interpretive algorithm, using Exner's (1974) comprehensive system. The reason for developing this computerized interpretive system is to augment the clinician's diagnostic understanding of the patient, to improve the decision making capabilities of the clinician, and to increase clinical efficiency.

In this paper, we will describe user's and system specifications, as well as the equipment configuration used to interpret Rorschach data. Finally, we will describe and report the results of a study of clinician's acceptance of computer-generated Rorschach narratives. It is our hope that this paper will help stimulate further developments in this interesting line of research.

\section{CONSTRUCTION OF AN ON-LINE SYSTEM}

\section{Background}

The decision to develop a computer-assisted interpretation method for the comprehensive Rorschach system was based on several factors. First, usage of Exner's (1974) system has increased greatly since its introduction (Klopfer \& Taulbee, 1976). Prior to the advent of the comprehensive system, some researchers had forecasted the demise of the Rorschach method as a clinically viable assessment tool (Knutson, 1972). Klopfer and Taulbee (1976) have suggested that the comprehensive system was instrumental in revitalizing interest in the Rorschach inkblots. Second, the system has integrated important features of other Rorschach systems, so that it approaches being a unitary Rorschach system. Third, the system emphasizes the structural nature of the projective data for analysis. Although the system considers the sequence of scores in the protocol and the content of the responses, the cornerstone of the analysis is the structure of the data. Fourth, the rules applied to the data have been extensively researched and supported. Although Exner (1974) warns against applying the comprehensive Rorschach system in a cookbook fashion, the system's well delineated interpretive rules and its systematic method of analysis provide the basis for the construction of the computer algorithm.

\section{Construction}

The development of the Rorschach algorithm began with the identification of interpretive rules for the ratios, percentages, and derivatives of the structural analysis. By using the normative data of the comprehensive system, normal and deviant ranges for the Rorschach variables were determined. Three interpretive statements for each rule, and in some cases for a group of rules, were generated. This method produced 150 rules with a dictionary of 400 statements. The reason for developing three statements for each rule and randomly choosing one for each report was to individualize the output and to avoid redundancy.

\section{Functional Specifications}

A trained clinician administers and scores the Rorschach protocol. However, the person assigned to enter the data into the computer system is likely to be a clerical-level person. This allows the clinician to devote more time to other aspects of the psychological evaluation. The user is seated in front of a cathode-ray tube (CRT) terminal connected to an interactive microcomputer system. All interactions with the computer are through the CRT. Once the user and the system are in an interactive mode, the user can perform two basic jobs: $\mathrm{He} / \mathrm{she}$ can create a new Rorschach data file or retrieve a stored data file. The user creates the data file by assigning the person tested an identification number that is then entered into the system. Upon opening the file, the user inputs pertinent background and demographic information. This information includes age, sex, ethnic background, educational and employment status, purpose of assessment, and level of cooperation. After these data are entered, they are presented on the screen for the user to inspect. If no errors are detected, the user informs the computer system that the data were correctly entered. This prompts the computer system to store these data in memory for eventual storage on a floppy disk. When errors are detected, the user makes the corrections before storing additional information. At this point, the user proceeds to enter the values of the variables that make up the standard structural summary of the comprehensive system. The stored information consists of determinants, content categories, location features, developmental quality, form quality, and special scorings. These data are used to calculate the ratios, percentages, and derivations that are needed for the structural analysis. When a frequency score for 1 of the 109 variables is zero, the user can skip to the next variable by pressing the "return key." This automatically assigns a default value of zero to the variable. Although the number of variables is large, the time required to enter the data into the computer system has averaged from 3 to $5 \mathrm{~min}$. When the data are correctly stored, the user presses the "Y" key in response to the question, "Were the data entered correctly $(\mathrm{Y} / \mathrm{N})$ ?" An affirmative response results in another question displayed on the video screen, "Would you like the data analyzed (Y/N)?" At this point, pressing the "Y" key initiates the analysis and permanent storage on a floppy disk. When the " $N$ " key is pressed, the data are stored on a disk without being analyzed. However, the analysis of the data can be conducted anytime later, at the discretion of the user.

\section{Systems Specifications}

The systems specifications describe the computer adaptation of the algorithm for on-line interpretive reporting. After the data have been stored in the computer system, the ratios, percentages, and derivations used in the structural analysis are computed. This infor- 
mation and other relevant features of the data are examined to determine which rules (in a prespecified file) apply to the data. The method of searching for rules and interpretive statements is designed to be consistent with the paragraph order of the report. For example, rules and their interpretive statements for cognitive processes are initially identified. The next paragraph considers the emotional state of the person, with subsequent paragraphs in the report handled in a similar fashion. One statement is randomly selected from the three alternates for each rule and sent to computer memory for temporary storage. Once the structural analysis has been completed, the string of statements is sent to the line printer.

\section{Equipment Specifications}

Computer equipment includes a DEC 11/03 microcomputer with $32 \mathrm{~K}$ memory, a VT100 CRT, a Diablo printer, and two floppy-disk drives. All programming was completed in multiuser BASIC programming language. In designing the software, one floppy disk was used for the algorithm and the other disk for the subject data. The algorithm disk contains the format for storing the data, the prespecified rules, the dictionary of interpretive statements, and the logic for calculating the variables of the structural analysis. The user's disk is designed to store the raw data of each Rorschach protocol in individual files. Retrieval of a subject's data file is thus readily accomplished by entering his/her identification number into the computer system. All tasks performed by the computer are in a single-job mode.

\section{Output}

Figure 1 shows an example of a computer-generated Rorschach report. As shown in Figure 1, the report begins with basic demographic information about the examinee and his/her level of cooperation during the assessment. The paragraph-formed narrative describes the person's cognitive processes, level of emotional stability, quality of interpersonal and inner life, and predominant defense mechanisms used. Based on the findings, a "possible" diagnosis is made.

\section{VALIDITY STUDY}

The clinical value of the computer-generated reports depends partially on whether clinicians view the reports as containing information relevant to the understanding and treatment of the individual. How well the reports meet these criteria is an indicator of the validity of the algorithm. To address this issue, we compared the computerized output with the report of an expert clinician.

\section{Method}

Three psychologists trained and experienced as psychodiagnosticians were asked to judge the clinical value of 10 sets of Rorschach reports. The reports were derived from examples given by Exner (1978). Each set of reports consisted of the structural interpretation used in the text and its computer-
A. B. is a 28-year-old single female. She reports completion of a 2-year junior college program and is currently employed as a teacher. Her level of cooperation during testing was excellent.

Individuals such as this respond to testing with an intellectual approach that is sufficient to the task. She makes use of an appropriate amount of details and generalities in response to the cards. She has the capability to be adaptive in her thinking. She tends to be an underachiever. Such individuals lack the ability to adequately test reality in their day-to-day operations. Under stress, her thinking becomes excessively rigid and constricted.

Individuals such as this have substantial psychological resources that are well organized and accessible. Such people are not able to control affective displays when appropriate. Labile emotions can be expected. Individuals such as this tend to avoid situations that are emotionally laden. She is apt to withdraw from the world under emotional pressure. This individual has significant feelings of helplessness but does have the capacity to cope.

This person is basically introverted and suffers from low selfesteem. There is an indication of self-introspection. These are unconventional people. Others of ten see them as negative, contrary, and oppositional.

Defense mechanisms include denial and intellectualization. The individual seems to be experiencing increasing defensive and adaptive failure.

Possible clinical symptoms include depression. The clinician should check for possible suicide intention.

Possible diagnosis is depressive reaction.

Figure 1. An example of a computer-generated Rorschach report.

generated counterpart. The computer-generated report was obtained by entering the data of the structural summary into the computer system for analy sis.

The instrumentation for this study was a 14-item agreedisagree questionnaire. The items of the questionnaire were designed to compare the reports on dimensions that many psychodiagnosticians consider basic to a clinically relevant report (Schafer, 1954; Weiner, 1972). Some of the dimensions used in the comparison included: (1) diagnostic understanding, (2) usefulness of information in clinical judgment, (3) meaningfulness of information for treatment planning, as well as other related areas. One item of the questionnaire asked whether "Report A provides a more meaningful interpretation of the individual's psychological state than Report B." To reduce the possibility of an order effect occurring in the judges' response patterns, the reports in each set were counterbalanced. For five of the sets, Report A referred to the computer-generated report; in the other five cases, it referred to the clinician-generated report. All reports were hand typed in the same format. Also, random ordering of the sets of reports was used to further prevent biased responding by the judges. However, for analysis purposes, the data were recorded in a single direction, to enable agreement among judges to be measured. Interjudge reliability was then computed, using the kappa technique (Cohen, 1960). This procedure yielded statistically significant kappa values for 9 of the 14 items. Table 1 lists the kappa values for each of the 14 dimensions used to compare the computer-generated and cliniciangenerated reports.

\section{Results}

For all observed decisions for the 10 sets of Rorschach reports, $k=.79$. This result was significantly greater than 
Table 1

Level of Interjudge Agreement on Computer-Generated Reports

\begin{tabular}{lc}
\hline \multicolumn{1}{c}{ Report Dimensions } & Kappa Values \\
\hline Psychological State & $.53 \dagger$ \\
Trait Characteristics & $.98 \dagger$ \\
Personality Structure & -.08 \\
Current Psychopathology & .21 \\
Adaptive Capabilities & $-.04^{*}$ \\
Interpersonal Characteristics & $.03^{*}$ \\
Cognitive Processes & .10 \\
Defense Operations & $.76 \dagger$ \\
Idiographic Information & .25 \\
Free of Contradictions & $.47^{* *}$ \\
Clarity/Conciseness & .34 \\
Diagnostic Understanding & $.59 \dagger$ \\
Treatment Planning & $.86 \dagger$ \\
Clinical Relevance Data & $.48 \dagger$ \\
Composite & $.79 \dagger$ \\
\hline
\end{tabular}

${ }^{*} p<.05 . \quad{ }^{*} p<.01 . \quad$ tp $<.001$.

zero $(p<.001)$. The judges agreed on seven report dimensions that the computer-generated narratives were comparable or superior to the clinician-generated reports. The computerized reports were judged as relatively free of contradictions $(p<.01)$ and as providing a clinically meaningful description of the person's psychological state, trait characteristics, and defensive operations. In addition, these reports were found useful in formulating both diagnostic impression and treatment plans. Accordingly, the judges also agreed that the computer-derived reports were clinically informative and valuable.

There were several areas in which negative or neutral findings occurred. The judges agreed that the computerized reports did not adequately describe the adaptive capabilities or interpersonal characteristics of the person $(p<.05)$. On several other report characteristics, the level of agreement among judges did not reach statistical significance. Lack of agreement was found for those dimensions that described the personality structure, the cognitive processes, the level of psychopathology. Judges also disagreed on the overall quality of the idiographic information and the clarity of the information in the computerized reports.

\section{Discussion}

Although these findings are preliminary, they suggest that the computer algorithm used to analyze the Rorschach data is able to generate reports that are useful and valid. They further indicate that the comprehensive Rorschach system is amenable to automation. There are, however, some indications that the algorithm does not provide clinicians with a full description of the person's personology. This problem, although central to clinicians' resistance to computer applications of psychological assessment tools, is not insurmountable. Modifying the computer algorithm to include the actual content of the projective data in the analysis and providing more idiographic information in the narrative should help with this.

The strengths of the computerized Rorschach narratives include the descriptions of the psychological state, trait characteristics, and defensive operations used. An equally important index of the algorithm's usefulness and validity is that the computerized reports were judged to contribute relevant information to the formulation of diagnostic impressions and treatment plans.

As expected, there were weaknesses in the computergenerated report. The judges reported that these reports were inadequate in their explanation of the person's adaptive capabilities and interpersonal qualities. In both cases, the judges felt that the information included in the reports was too vague. The lack of concise information in these areas contributed to the nonsignificant kappa values for the idiographic information and personality structure dimensions. Similarly, the cognitive process descriptions in the reports were cited by two judges as ambiguous and redundant. These weak areas in the report were responsible for the low level of agreement among the judges on the clarity/conciseness dimension.

The findings of this study indicate that the computer algorithm used to process Rorschach data is capable of generating clinically relevant reports. Although the computerized report has several problems, refinement and expansion of the decision rules and interpretive statement should eliminate these weaknesses.

\section{REFERENCES}

Cohen, J. A coefficient of agreement for nominal scales. Educational and Psychological Measurement, 1960, $20,37-46$.

Exner, J. E., JR. The Rorschach: A comprehensive system (Vol. 1). New York: Wiley, 1974.

EXnen, J. E., JR. How clinicians use the Rorschach. Journal of Personality Assessment, 1976, 36, 403-408.

EXnER, J. E., JR. The Rorschach: A comprehensive system (Vol. 2). New York: Wiley, 1978.

GorнАм, D. R. Validity and reliability studies of a computerbased scoring system for inkblot responses. Journal of Consulting Psychology, 1967, 31, 66-70.

Klopfer, W. G., \& Taulbee, E. S. Projective tests. In M. R. Rosenzweig \& L. W. Porter (Eds.), Annual review of psychology. Palo Alto, Calif: Annual Review, 1976.

KnUtson, J. R. Rorschach. In O. K. Buros (Ed.), Seventh mental measurement yearbook (Vol. 1). Highland Park, N.J: Gryphon, 1972.

Piotrowski, Z. A. Perceptanalysis. Philadelphia: Ex Libris, 1974.

Plotrowski, Z. A. CPR: The psychological $X$-ray in mental disorders. In J. B. Sidowski, J. H. Johnson, \& T. A. Williams (Eds.), Technology in mental health care delivery systems. Norwood, N.J: Ablex, 1980.

Schafen, R. Psychoanalytic interpretation in Rorschach testing. New York: Grune \& Stratton, 1954.

SmITH, M. S. The computer and the TAT. Journal of School Psychology, 1968, 6, 206-214.

VELDMAN, D. J. Computer-based sentence completion interviews. Journal of Counseling Psychology, 1967, 14, 153-157.

Veldman, D. J., Menaker, S. L., \& Peck, R. F. Computer scoring of sentence data. Behavioral Science, 1969, 14, S01-507.

WEINER, I. B. Does psychodiagnosis have a future? Journal of Personality Assessment, 1972, 36, 534-546. 